\title{
Survey: energy efficient protocols using radio scheduling in wireless sensor network
}

\author{
Deepa Mathew K., Anita Jones \\ Electronics and Communication Engineering Department, Karunya University, Coimbature, India
}

\begin{tabular}{l}
\hline \hline Article Info \\
\hline Article history: \\
Received Aug 6, 2019 \\
Revised Oct 12, 2019 \\
Accepted Oct 22, 2019 \\
\hline
\end{tabular}

\section{Keywords:}

Duty cycle

Energy conservation

Network lifetime

Radio scheduling

WSNs

\begin{abstract}
An efficient energy management scheme is crucial factor for design and implementation of any sensor network. Almost all sensor networks are structured with numerous small sized, low cost sensor devices which are scattered over the large area. To improvise the network performance by high throughput with minimum energy consumption, an energy efficient radio scheduling MAC protocol is effective solution, since MAC layer has the capability to collaborate with distributed wireless networks. The present survey study provides relevant research work towards radio scheduling mechanism in the design of energy efficient wireless sensor networks (WSNs). The various radio scheduling protocols are exist in the literature, which has some limitations. Therefore, it is require developing a new energy efficient radio scheduling protocol to perform multi tasks with minimum energy consumption (e.g. data transmission). The most of research studies paying more attention towards to enhance the overall network lifetime with the aim of using energy efficient scheduling protocol. In that context, this survey study overviews the different categories of MAC based radio scheduling protocols and those protocols are measured by evaluating their data transmission capability, energy efficiency, and network performance. With the extensive analysis of existing works, many research challenges are stated. Also provides future directions for new WSN design at the end of this survey.

Copyright $(0) 2020$ Institute of Advanced Engineering and Science. All rights reserved.
\end{abstract}

\section{Corresponding Author:}

Deepa Mathew K,.

Electronics and Communication Engineering Department,

Karunya University, Coimbature, India.

Email: deepamathewk.ec@gmail.com

\section{INTRODUCTION}

The Wireless Sensor Network (WSN) significance is not only limited to the design of a stand-alone application, whereas today it is a sub-system of the internet of things (IoT) [1]. The IoT brings scalability into the WSN by connecting WSN nodes through gateways [2,3]. The issue of enhancing network lifetime is always a critical requirement, whether the application is standalone WSN or an IoT application [4]. In order to improvise the network overhead, various energy management approaches are proposed at different layers of WSN [5]. The energy efficient congestion control and routing protocols are designed at the transport and network layer respectively [6,7]. At the physical layer, radio schedules, duty cycle, used of directional antenna, etc. are the approaches towards optimizing the consumption of energy [8]. The traditional approach of managing duty cycle using IEEE 802.15.4 standard is well accepted for IoT also due to its low energy consumption [9]. The energy conservation scheduling algorithms are broadly classified into two categories namely centralized and de-centralized [10]. In a long duration transmission, the signal experiences a degraded channel condition that causes excessive use of energy. The use of time division multiple access (TDMA) protocol ensures optimal use of energy [11]. The parameters while designing scheduling schemes need to consider the type of sensors, network topology, deployment policy, sensing area, transmission range, 
time synchronization, localization, etc. [12]. The optimal goal of designing energy efficient scheduling schemes is to enhance the network lifetime.

The radio in the communication module of a sensor node has three different states: 1) Active-State, 2) Idle-State, and 3) Sleep-State. The radio scheduling scheme requires designing time synchronization among these states [13]. The highest amount of energy is consumed while the active state of radio because during this state both transmission and receiving of the signal takes place, whereas in idle state the radio is on, but the communication process doesn't take place, so the energy consumed during this state is waste. The automation of switchover between idles to sleep and sleep to active as well active to sleep is the core requirement of radio scheduling [14]. The radio energy consumption $\left(\mathrm{R}_{\text {Energy }}\right)$ can be formulated as [15].

$$
R_{\text {Energy }}=\left[\left(c . P_{t x}\right)+b\right] T
$$

Where $\mathrm{C} \rightarrow$ coefficient of energy transmission, $\mathrm{b} \rightarrow$ energy constant, $\mathrm{P}_{\mathrm{tx}} \rightarrow$ energy exploit in signal transmission and $\mathrm{T} \rightarrow$ total transmission time.

The radio scheduling mechanism for sensor networks is the key factor for energy conservation after the network is deployed. The all the sensor nodes are in sleep mode, the node cannot perform sensing and communication task and consumes very low energy. Consequently, the key goal of energy scheduling management scheme is to keep minimum number of sensor devices into active-mode for the necessary task and put other sensor nodes in the sleeping mode to conserve the energy. The advantage of scheduling a radio to sleep stage, it saves energy significantly as it operates at a low duty cycle, in turn, enhances the overall lifetime of the network and other quality of service parameters. In addition, the existing research on radio scheduling schemes mainly focused on single node sensor module, while few studies given attention towards the sensed module scheduling for multisensory nodes. The prior research considers the radio of each node as the module which consumes the most energy.

However, with the development of wireless sensor technology, sensors in the networks are integrated with number of sensor nodes, and those can bring different sensing capabilities, for example; temperature measurement, distance prediction, and so on. Through the sensing observations, active sensor nodes can coordinate with each other and track the targeting task. The multi-sensory approach achieves good tracking results than single sensor sensing observation. Thus, sensor scheduling management scheme is taken into the account which balance the energy consumption rate as well as transmission and storage related problems. As an example; video sensory nodes consumes high energy with more bandwidth while multimedia transmission, that is the major factor in the network performance. Therefore, low energy consumption during scheduling management is the higher priority in designing a WSNs. To prolong the network lifespan, sensor nodes are often scheduled at sleeping mode. While neighboring nodes performs the common sensing task and all other nodes are not required to perform the same task in the entire network lifetime. In order to improve the sensor energy utilization and prolong the network lifetime, a distributed cluster-based scheduling algorithm is introduced by Kannan et al. [16]. This approach significantly achieved the 7.5 to 12 percent accuracy in the overall energy saving as compared to existing hierarchical protocols.

Furthermore, a sleep scheduling mechanism is broadly adopted technique to balance the network performance by consuming minimum energy. A distributed sleep scheduling scheme allows the sensor node to completely covers the sensing areas and turn off the node if the communication doesn't take place or else do not have enough energy [17]. Lui et al. [18] have proposed a joint energy efficient routing and scheduling protocol which provided possibilities for evaluating the performance of the existing protocol and serves as a benchmark to evaluated real-time heuristics which endeavor to enhance the WSN lifetime. In traditional sleep scheduling, the sensor nodes perform numerous tasks with an unspecified time and consume external energy. Therefore, to overcome this problem, an interference-free TDMS sleep-scheduling mechanism is introduced which avoids passive listening, collision, and overhearing [19].

The present survey study is mainly focusing on the significance of radio scheduling mechanism which improves the network lifetime by minimum energy consumption. However, the maximum energy saving can be attained at physical layer, whereas Medium Access Control (MAC) layer responsible to manage the radio activities, in the result more energy can be saved. An efficient MAC protocol uses the radio scheduling scheme as a result saves the more amount of energy at every level. Hence the MAC protocol significantly fulfill the requirement of designing an energy efficient scheduling scheme for wireless networks.

Therefore, the survey study takes advantages of prior research works of energy efficient radio scheduling for WSN deployment and mainly focused on maximizing the overall network lifetime. The purpose of this survey study is to investigate the efficiency of radio scheduling for energy conservation with respect to existing research work. Also, overviews on different MAC protocols with respect to scheduling mechanisms help to enhance the sensor networks lifetime. The structure of the comprehensive

Survey: energy efficient protocols using radio scheduling in wireless sensor network (Deepa Mathew K.) 
survey study can be organized as; Section-II illustrates the different energy efficient radio scheduling schemes for WSNs. Section-III briefly studies on related work. Section-IV provides Research challenges towards designing an energy efficient WSNs using radio scheduling. Section-V ends with the conclusion of the survey study.

\section{MECHANISM OF RADIO SCHEDULING FOR ENERGY CONSERVATION OVER WSN}

The superiority of MAC protocol is directly related to the performance of WSNs because MAC can control the sensor nodes accessing the medium. The various existing radio scheduling mechanism has been introduced to manage the scheduling operation for energy conservation. However, different MAC protocols are broadly classified as; i) Contention-based protocols and ii) Contention free protocols. The detail description about these protocols are given as follows.

\subsection{Contention-based" MAC protocols}

\subsubsection{Sensor-MAC}

Sensor-MAC protocol is introduced by Ye et al. [20, 21]. It is energy efficient MAC protocol specifically designed for sensor networks. The core objective is more energy saving by maintaining scalability. The Sensor-MAC protocol introduces a cyclic active approach and sleep-scheduling scheme to setup the low-duty cycle operation at every sensor. According to this operation every sensor node is timely moved into sleep-state and then wake-up and enters into active-state for communication with other sensors if it is needed. In sleep-state, the radio is completely turned off, and the timer is set up to awake the sensor at a later time. The periodic cycle of sleep and active-state is referred as a frame. The following Figure 1 illustrates scheduling mechanism of sleep and an active state in S-MAC protocol.

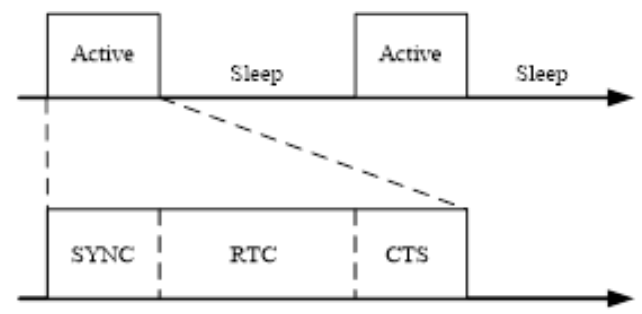

Figure 1. Active and sleep scheduling in S-MAC protocol

A duty-cycle is a ratio between the predefined active time and total time of that period [21]. The active state is divided into time intervals for sending or receiving the packets e.g. SYNC, RTC,CTC packets. The active time is predefined on the basis of physical-layer \& MAC-layer parameters (e.g., bandwidth and frame-size). While the sleep-mode timing is updated as per the requirement of different applications, that changes the actual duty cycle. S-MAC protocol is more energy efficient than 802.11. It is simple to implement; long term communication can be performed using a message passing approach. Nevertheless, due to the specified active or sleeping time ratio, some amount of bandwidth is un-usable, and delay is very high. The major drawback is high volume of data delivery because Sensor-MAC is developed to optimize the latency with more energy conservation.

\subsubsection{Demand sleep-MAC protocol: DS-MAC:}

It performs a dynamic duty cycle with the aim of high tradeoff among minimum latency and more energy conservation with less overhead. It introduced a mechanism like demand sleep mechanism, which responsible to manage the sensors sleeping-time based on total receive packets. When the scheduled time period is beyond the threshold value, DS-MAC shortens the sensor sleeping time, because switching of the sleep state to active state consumes much energy [22]. If the amount of received packets are lesser than the threshold value, sensors increases its sleep mode time to save the maximum energy which is wasted in idle-state. In this protocol, every sensor node exploits SYNC packets to setup and handle the clocksynchronization as similar as Sensor-MAC. Though, DS-MAC protocol adopts duty-cycle mechanism for reducing the noise problems. In this protocol duty, cycling is dynamic; it is not fixed. Asynchronous MAC protocols example; Berkeley-MAC, Cross-MAC and Receiver-Initiated MAC introduced for energy harvesting WNSs [23]. The experimental results illustrated that RI-MAC protocol performs better in energy conservation. Additionally, these protocols support individual duty cycles. To design a more efficient 
DS-MAC protocol, some additional parameters need to be defined, like example; delay and duty-cycle in each synchronization packet. The drawback is, every sensor needs to balance its energy consumption rate, which needs more storage space and computation overhead

\subsubsection{Directional-MAC protocol:}

The Directional-MAC protocol is an efficient protocol with respect to optimal latency with low energy consumption [24-25], adopted for data aggregation. Authors addressed packet transmission challenge in multihop network. The core objective was to attain energy conservation and minimum latency. To enable continuous packet transmission over multihop routing, D-MAC staggers the sensors schedule on the multihop route and allows the sensors to activate serially. During scheduling operation, the time slot is divided into three states, i.e. sending, receiving and sleeping. In sending time, a sensor tries to forward a data packet to next hop and receive an acknowledge packet. In receiving time, the sensor expecting to receive the packet and send acknowledge back to the sender. D-MAC is developed to solve the overhearing problems by giving sleep-schedule of sensor an offset which depends on its novel method.

Additionally, the duty-cycle setup was made on the basis of traffic load requirement in the sensornetwork. Kumar et al. [26] introduced a traffic load adaptive algorithm for varying inherent traffic scenarios in the WSNs. In [27] Wang et al. have presented a comprehensive survey study on D-MAC protocols for adhoc sensor networks. Authors believed that this survey study provides a benchmark for the development of improved D-MAC protocol for WSNs applications. Also highlighted major research issues for D-MAC protocols for WSNs and expected that new D-MAC protocol design leverages on existing D-MAC protocols. Yi et al. [28] addressed the issues of long term communication capabilities over Ad-hoc networks using D-MAC protocols and designed a framework of the long-term communication protocol in Ad-Hoc networks.

\subsubsection{TimeOut-MAC:}

TimeOut-MAC protocol is an energy-efficient protocol, derived from S-MAC protocol for WSNs. The essential goal is to minimize the idle-state time duration by defining dynamic duty-cycle and forward all data packets in the burst of variable length inactive state, and there is a gap between sleeping time called bursts [29]. To balance an active-mode time period during high traffic load, dynamically TimeOut-MAC setup the threshold value. This reduces idle listening. The node activates periodically and communicates with neighboring nodes. It uses RTS and CTS, ACK mechanism which avoids collision with reliable transmission. The node enters into listening-mode and performs data transmission under active mode. If no event performs in active-mode at a specified threshold (Th), then active mode switch off and automatically node goes to sleep mode. At the period of continuous data, transmission nodes communicate continuously without entering sleeping mode. The following Figure 2 pictorially represents the scheduling mechanism of the T-MAC protocol.

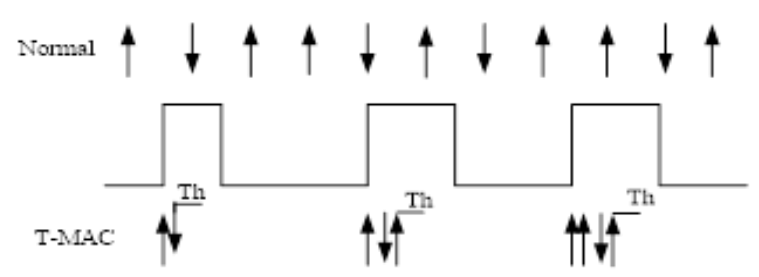

Figure 2. Scheduling in T-MAC protocol

The experimental results represent that both S-MAC and T-MAC protocols achieve up to $98 \%$ energy consumption reduction as compared to other protocols. The disadvantage of this protocol is an early sleep problem where a node goes to sleep mode even if it's neighboring node forward some data to it. Singh et al. [30] introduced new T-MAC protocol with new features called power saving mode which makes the T-MAC more energy efficient. The additional time duration or active time is not considered when no activity is performed, and leftover energy of specific sensor-node gets below with certain threshold time, then its activation time-out is minimized by 10 percentage. This minimizes the wastage of energy in the idle state and improves the network overhead. In [31] authors presented time efficient sleep-scheduling mechanism for underwater WSNs. The essential factor was time conservation without considering the delay in the sensors having no packet delivery to its parent node. Hence, data forwarding is very faster than existing sleep scheduling schemes prevent the wastage of time. 


\subsubsection{Contention-free MAC protocols}

The theoretical study of contention free MAC protocol is presented by Busch et al. [32] which able to bring the network from arbitrary position to collision-free position. Because it is a distributed protocol, the stabilization collisions are unavoidable. Examples of contention free MAC protocols are Traffic adaptive medium access protocol, self-organizing MAC protocol, distributed energy consumption MAC protocol and contention free scheduling time division medium access MAC protocol. The detailed study of each protocol is discussed as follows.

\subsubsection{Traffic adaptive medium access protocol TA-MAC}

It is a TDMA based protocol which offers efficient energy channel access without any collision for wireless networks. Through this, optimal energy is achieved by guaranteeing non-collision data transmission and allows sensors to switch idle-state with minimum energy usage. TA-MAC assumes a single channel is slotted for data as well as signal transmission. The time slot is mapped in a sequence of random access (RAcc) time (i.e., signaling slot) and schedule-access (SAcc) time (i.e., transmission slot). The TA-MAC protocol begins with RAcc time where every sensor node is randomly select the time slot and later transmits the data. The duty-cycle of RAcc time and SAcc time depend upon the nature of the network. However, the slots are utilized for data-transmission and interchange the traffic aware scheduled time information among the sensors. The sensor has to provide its schedule information through schedule packet exploiting schedule exchange protocol before transmission of actual data. Periodically, the schedule exchange protocol updates the scheduling information and maintains this information between the neighboring nodes. The advantage of TA-MAC protocol achieves more energy conservation owing to the high percentage of sleep state. Also, it achieves higher throughput as compared to the contention based MAC protocols.

\subsubsection{Self-organizing MAC protocol: SMAC}

It is a distributed, contention free MAC protocol developed for sensor networks [33]. It enables a group of sensors to create a route and establish a schedule for communication with neighboring nodes without any local/global master node. In this protocol, every node is capable of setting its radio schedule (i.e., on/off) and adjust the carrier frequency for different bands. The set of available bands are relatively large. Additionally, the require information about sensor-node in the radio networks is utilized to schedule the duty-cycle over the network. Every sensor manage the super-frame, by which schedule the communication time slots of sensor nodes. With the specified time-slot, every sensor node can collaborate with their neighborhood nodes. However, there is possibility of time-collisions between the slots due to the unknown channel assignment. To resolve such collision problems, every node in the network operate with their own frequency range which is arbitrarily considered from radio frequencies. Once the link established the node schedule the turn-on time of transceiver for further communication and automatically turns-off if there is no collaboration between the nodes. From this mechanism, can achieve efficient energy saving at every node. The major drawback of SMAC protocol is low bandwidth usage, e.g., if the sensor needs to forward a data-packet to its neighbor, it cannot be re-utilized the same time slot of other neighbor nodes.

\subsubsection{Distributed energy consumption MAC protocol: DE-MAC}

In this protocol network is structured with large sensor-nodes and randomly distributed over the geographical region. During the transmission process, DE-MAC uses sensor's information from its neighboring node and creates number of cluster sets including cluster-head $(\mathrm{CH})$ as well as base station (BS). The $\mathrm{CH}$ responsible to aggregate the data and transfer to the BS. However, the DE-MAC protocol is scheduling based protocol where operations are categorized into two specific communication groups such as inter cluster and intra cluster communication. While the time of cluster construction, sensor-node determines the energy level with respect to network capability. Likewise TA-MAC, this protocol contains two distinct time-slots, one is RAcc and SAcc. Furthermore, each slot is again splitted for data partition and access control. The sensor-node which is assigned with time slot uses the access control to broadcast the receiver identification. The identified receiver node keeps on and remaining nodes stay on sleep-mode with minimum power consumption. However, Sefuba et.al presented energy efficient dynamic MAC protocol for clustering based WSNs. The study adopted a cross-layer cluster scheduling approach which mitigates the idle-listening problems as well as reduces the packet overhead. The transceiver node in the proposed model transmission process involves three major operations viz; sleep-state, active-state and back-off operation. Also presented a cooperative communication system among base-station and clusters. The simulation outcomes represented that performance of energy consumption, throughput, as well as delay in the sensor networks. The overall accuracy is very good as compared to existing frameworks. 


\subsubsection{Contention-free scheduling TDMA-MAC protocol: CS-TDMA-MAC}

Similarly, it is also a contention free TDMA based protocol. In this periodic messaging system perform a message scheduler which enables transmitting and receiving the node messages and ensures that the transmission medium is contention free. Initially, a set of contention-free periodic messages are generated from message attribute assignment and later set off a periodic task is constructed through a given set of the contention-free periodic message by manipulating each message attributes to task attributes. Because message scheduler is a contention-free, all sensors devices require to setup the message of its own. Thus, the complexity of sensor-node increases with message transmission and received from that sensor, rather than length of all messages in the network. This highly reduces the storage and time complexity of network scheduler and thus results in computation, memory and energy conservation. Hence, this type of MAC protocol is efficiently scalable for large scale sensor networks

\subsection{Hybrid protocols}

Hybrid protocols contain both contention-based and contention-free protocols features, and those are introduced as follows:

\subsubsection{Hybrid CSMA and TDMA MAC protocol}

It is a hybrid MAC protocol, where CSMA offers a simple and distributed scheme for adaptive traffic and TDMA provides the centralized and scheduled scheme which targets the quality of services by resource assignment [34]. In this, all sensors contain two communication channels viz; data channel and control channel. TDMA protocols perform on the data channel for periodic transmission, and CSMA protocol is utilized for signal traffic transmission. In CSMA delay increases rapidly with increasing traffic load, which may lead to complexities in real-time orbit control. While in TDMA, a fixed time slot based TDMA network defines packet delay and need accurate duty cycling. As comparing the CSMA protocol, TDMA maximizes the communication time during network traffic is lower than the threshold. The hybrid CSMA and TDMA MAC protocol have been applied in many applicatory areas viz; Ad-hoc vehicular networks [35], Tree-MAC [36], and MCL-MAC [37] and many more. These protocols have been offered to achieve increase throughput with minimum control overhead. The frame structure of the hybrid CSMA and TDMA MAC protocol is presented in Figure 3.

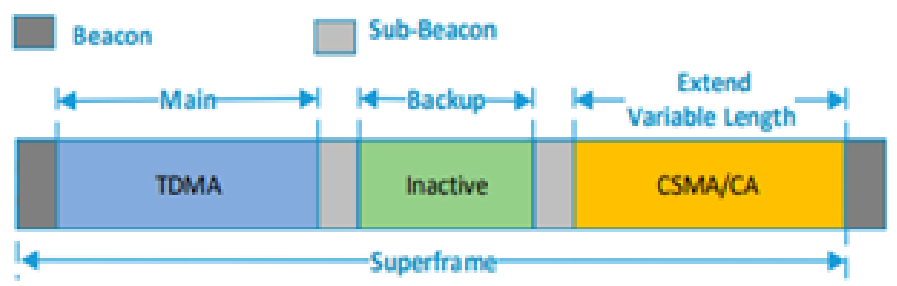

Figure 3. Structure of hybrid CSMA and TDMA MAC protocol [34].

The superframe of the hybrid protocol contains three cyclic periods (i.e., TDMA, Inactive and CSMA). Additionally, beacons are utilized for signal transmission, and there is no data transmission during the inactive period. With the new channel assessment scheme and adaptive scheduling system, the hybrid CSMA and TDMA protocol are expected to enhance the network lifetime by energy conservation [34]. The average energy consumption during packet transmission, receiving an idle state can be expressed as

$$
E_{C S M A / T D M A}=\frac{n_{B} E_{i d}+n_{C} E_{r x}+\left(1-p_{f a i l}\right)\left(E_{i d}+E_{r x}\right)+L E_{t x}}{n_{B}+n_{C}+(2+L)\left(1-p_{f a i l}\right)}
$$

$\begin{array}{lll}E_{C S M A / T D M A} & : & \text { Total power consumption } \\ \mathrm{n}_{\mathrm{B}}, \mathrm{n}_{\mathrm{C}} & : & \text { No. of slots required in back off and clear channel assessment for each attempt } \\ \mathrm{E}_{\mathrm{tx}}, \mathrm{E}_{\mathrm{rx}}, \mathrm{E}_{\mathrm{id}} & : & \text { Average energy consumed during transmission, receiving, and idle state } \\ \mathrm{P}_{\text {fail }} & : & \text { Packet fail } \\ \mathrm{L} & : & \text { Occupied length }\end{array}$




\subsubsection{Zebra-MAC protocol: Z-MAC}

Similarly, Z-MAC is also a hybrid protocol is introduced by [38] which integrates the TDMA and CSMA strengths while offsetting their weaknesses. The important characteristic of this hybrid protocol is dynamically adapts the network contention level. The low-contention level, behaves like a CSMA and achieves low latency and high channel utilization. In high contention level, it's like TDMA and reduces collisions between two neighboring hops at very low cost and achieves high channel utilization. The investigated Z-MAC protocol capable of minimizing the energy consumption rate over the sensor network and robust to dynamic network structure changes is the significant feature. From this feature can reduce the protocol to synchronization

\section{RELATED WORK}

From the past decades, many researchers paying more attention to WSNs design with minimum energy consumption problems by introducing different routing schemes and MAC layer protocols to increase the energy rate in WSN. The present comprehensive survey study reveals that the evolution of various energy efficient protocols was designed based on network topology (i.e., hierarchical, location-based routing... etc.). This section illustrates the existing work carried out on energy-efficient protocols and their problems. The critical analysis of the most popular existing techniques is presented.

Energy conservation is the significant factor in enhancing the overall network lifetime, and it is considered as a primary challenge in WSNs. Several scheduling mechanisms have been introduced with the goal of minimum energy consumption in the result can prolong the network-lifetime. The prior research on WSN scheduling majorly focused on network equipped with single sensor node and maintain the sleep scheduling time. A multi-sensor scheduling approach is investigated by Feng and Zhao [39] which considered the scheduling for both communication module and multi-sensor module. The WSN is organized in clustering format where each cluster head adaptively assigns a sleeping time for its cluster nodes according to the position of remaining nodes.

Another efficient sleep-scheduling mechanism for WSNs is introduced by Wan et al. [40]. This approach schedules the nodes into sleep or active mode for efficient energy consumption. Initially, the optimal radius is approximated to deploy all sensory nodes into clusters to balance the energy level. Secondly, based on packet collected by neighboring nodes, a fuzzy-matrix is generated which able to compute the similarity features and fuzzy theory is responsible to classify the sensor-nodes into multiple groups. The energy consumed during data-aggregation by cluster head is defined as;

Eagg $(1, d) \rightarrow$ lEDA

Where 1 is no. Bit packets, $d$ is distance, and EDA is energy consumption for data fusion per unit.

Another novel hybrid TDMA-CSMA-MAC protocol [41] aims to reduce the energy consumption in WSN. The TDMA scheme is responsible for handling the traffic burst problems. Furthermore, an adjustable transmission energy control mechanism is proposed which set up the different slots for different transmitting energy values for cluster heads and sensor nodes. The performance analysis of the proposed methods is evaluated by measuring throughput and total energy consumption. The total energy consumption includes energy transmission cost on sensor node to cluster head, and it is represented as follows.

$$
E_{\text {total }} \rightarrow E_{S c}+E_{C C} \rightarrow \sum_{i=1}^{n_{S}}\left(E_{i C P}+E_{i T P}\right)+E_{C C}
$$

Where $E_{\mathrm{SC}}$ and $\mathrm{E}_{\mathrm{CC}}$ are the energy transmission cost of the sensor node and cluster heads, $\mathrm{E}_{\mathrm{iCP}}$ and $\mathrm{E}_{\mathrm{iTP}}$ represent energy consumed in contention period and TA-TDMA periods of the sensor node to cluster head of ith nodes in WSN.

The few survey study of sleep scheduling mechanism is summarized in the study of Zhang et al. [42] and observed that most of the studies focused on asynchronous scheduling mechanisms. Moreover, the machine learning approach is widely applied in this field. Another survey of scheduling mechanism is proposed for WSNs security [43], where authors studied different scheduling scheme and presented their advantages and drawbacks. Additionally, the authors proposed a dynamic priority based multilevel packet scheduling method with the bit-rate classification which helps to minimize the end-to-end delay. The network security can be achieved by implementation of the RC-6 security algorithm during data transmission and receiving operation.

For large scale commercial WSNs, few sensors topology is too critical to maintaining. The existing research on network control with critical nodes specifically focused on network routing inside the cluster. It is non-trivial to manage the cluster connectivity without considering the critical nodes in the cluster based 
large scale commercial WSNs. Hence sleep schedule is the best approach to conserve the high amount of energy of sensor nodes in large scale WSNs. In [44] authors focused on priority based sleep scheduling for cluster-based commercial WSNs to save network energy and prolong the WSNs lifetime. A sleep or awake scheduling scheme for WSNs is introduced [45]. The aim was to conserve more energy without using duty cycling and improve the network lifetime as long as possible. The other existing energy efficient scheduling schemes are based on duty cycling techniques which takes more effort to improve their performance. Self-Adaptive sleep-awake scheduling is the best approach which uses game theory and reinforcement learning mechanism.

MAC protocol with joint routing mechanism is investigated [46] which reduces the transmission delay in WSNs. Due to increasing growth in the WSN application; there is a requirement for an optimized protocol for specific WSNs application. The proposed joint routing mechanism allows the nodes to schedule the packet transmission in the same duty cycle. It partitions the sensor nodes into disjoint sets to minimize the idle listening time of the node in the cycle without affecting network connectivity. Another energy efficient routing and joint duty-cycle scheduling method are investigated [47]. In this study, the author discussed how the nodes could be scheduled for sleep/awake in order to save energy during idle listening.

Additionally, the game theory approach is applied to evaluate the sleep or awake scheduling time, and energy efficient routing algorithm is introduced which makes routing decisions for each sensor. The sensor chooses an active node with high residual energy between the clusters. The Q learning approach is adopted for duty-cycle scheduling. An energy efficient sleep scheduling scheme is proposed [48] for wireless powered communication networks. The aim is to extend the wireless networks lifetime by energy harvesting approach.

An opportunistic routing mechanism with improved sleep scheduling method is proposed [49] which improve the WNSs lifetime with minimum energy consumption. For minimum energy consumption, authors adopted different routing approaches which are standard and improvised. The proposed opportunistic energy efficient routing strategy with sleep scheduling method offered high performance and improved the routing quality in WSNs. One more approach of joint routing scheme with radio scheduling mechanism is proposed by Buratti and Verdone [50] for centralized WSNs. Authors proposed two algorithmic approaches i) sequential algorithm and ii) joint scheduling and routing algorithm. The first algorithm responsible for reduces the maximum latency with minimum packet loss. The second algorithm combines the scheduling and path selection policies and solves the problems of no packet loss. Additionally, the study utilized Dijkstra's and graph coloring algorithm for path selection and analyzed the performance analysis based on the throughput ratio between the numbers of nodes.

An adaptive energy-efficient duty cycle management scheme improved the network delay performance and addressed the nodes selection duty cycle based on the energy consumption rate [51]. The experimental analysis evaluated the data load according to its energy consumption rate and shown that nodes in far sink distance have large duty-cycle. Whereas, nodes in closer sink distance have short dutycycle. Furthermore, the study represented equation models among duty-cycle, delay, and energy usage, which evaluated the nodes best duty-cycle. This approach can outperform as compared to existing schemes and provides better network lifetime. Duty cycled MAC protocols can improvise the sensor networks lifetime. However, most of the existing MAC protocols still suffer from overhearing and idle listening problems. To overcome these problems Oller et al. [52] investigated a novel wake-up radio scheduling scheme which reduces the idle listening and overhearing problems by switching off the nodes. In the simulation, authors considered time and energy consumption values and evaluated different nodes lifetime. In the previous study [53] similar wake-up radio scheduling approach is applied for traditional WSNs and shown it outperforms in terms of energy efficiency. The authors introduce the different MAC scheduler mechanisms with the aim to minimize the energy consumption rate in WSN [54-56].

\section{RESEARCH CHALLENGES}

By analyzing the prior research studies, can identify the many challenges in energy efficient radio scheduling scheme. Especially, the quality of service may not be provided. This could be illustrated by considering the network performance with the lowest channel quality containing more priority applications. Parallel data transmission technique is attracting in many WSN platforms emerged with scheduling support. The radio energy in WSN is limited, and thus it is require to device multi-channel MAC-protocols to manage traffic load, or support multitasks. The next generation network technologies need efficient scheduling scheme with multi-channel supportive MAC protocols to improve the communication quality with minimum energy consumption. Energy conservation by keeping the nodes into sleep state periodically is a fundamental approach for WNS platform. The highly distributed WSN platform contains small, lightweight sensor nodes. 
Radio scheduling is a significant mechanism in WSN to maintain packet scheduling and conserve the high amount of energy. Existing scheduling protocols used duty cycling method.

Though several MAC-based radio scheduling protocols has been investigated, and those have specific features. The reason behind is that sensor-networks are application specific and MAC layer protocols are application dependent. Generally, TDMS/CSMA are MAC family protocols which are collision-free nature and efficiently conserve the high amount of energy under high traffic condition. However, it has lower throughput and high delay rate in lower traffic condition owing to passive listening.

Additionally, TDMA-MAC protocol requires fixed-time synchronization among neighboring sensor nodes and has less accessibility as well as limited scalability to the dynamic network. Whereas, CSMA is contention-based MAC protocols which results in minimum energy consumption with the high delay in large traffic condition, but can reduce the delay with high-throughput under less traffic load. Based on network environment, energy-efficient MAC protocols can incorporate TDMA with other protocols to perform various requirements. Thus, to improve the energy efficiency level, there is a trend to consider joint scheduling energy-efficient MAC protocols for multilayer WSN applications, which offers multiple opportunities in the future WSN application design.

The dynamic energy management is another type of MAC layer scheduling scheme, which can achieve more energy conservation and enhances the sensor node lifetime. This mechanism does not affect the network performance and directly deals with node transition state in an energy efficient manner. The core point is to turnoff the sensor-node when no transition occurs and active them when needed. Such an eventbased energy management scheme is critical to achieve improve the node lifetime.

In CSMA protocol, sensor nodes transmit the data packets at the maximum energy level, saves all interfering sensor nodes from self transmissions. This protocol allows only single transmission at a time since all sensors are fixed within the pre-defined carrier sensing region. Thus, interfering nodes are not allowed to transmit data packets. This protocol has transmission complexities from a single layer viewpoint, which is not efficient. Therefore, there is a requirement of multilayer packet transmission with minimum energyconsumption.

Compared with IEEE 802.11 and energy aware MAC-based signaling approach, Sensor-MAC doesn't uses channel signaling mechanism, whereas, energy aware MAC-based signaling protocol need two independent channel nodes (i.e., transmitter and receiver). Additionally, energy aware MAC-based signaling protocol doesn't conserve the high energy during the idle listening mode. Therefore, Sensor-MAC protocol achieves high energy conservation by avoiding overhearing and efficiently transmits the large data packets. Hence, this protocol can be applicable for WSN applications.

\section{CONCLUSION}

The survey study overview the most recent research work in the state of art of energy efficient radio scheduling protocol design for WSNs. The scheduling protocols for WSNs are broadly categorized as; i) "Contention-based" MAC-protocols and ii) "Contention-free" MAC-protocols. The core idea behind this is to investigate the significant research trend energy efficient radio scheduling protocols based on techniques is employed. The study shows that energy conservation is considered as the essential parameter to enhance the WSN lifetime. In this comprehensive survey study have mainly focused on investigation of potential energy sources and the research efforts on their utilization. A network-wide energy efficient protocol can better manage its operation while taking into account the nodes' supply and energy consumption. Therefore, we recommend to the research community to consider both, the energy supply as well as the energy consumption in parallel while designing an energy efficient algorithm.

\section{REFERENCES}

[1] Y. Kuo, C. Li, J. Jhang and S. Lin, "Design of a Wireless Sensor Network-Based IoT Platform for Wide Area and Heterogeneous Applications," in IEEE Sensors Journal, vol. 18, no. 12, pp. 5187-5197, 15 June 15, 2018.

[2] M.Vo, N. Do, V. Tran, Q. Ma, C. Le and L. Mai, "A multi-storey building actuator and sensor system using 6LOWPAN based Internet of Things: Practical design and implementation," 2018 2nd International Conference on Recent Advances in Signal Processing, Telecommunications \& Computing (SigTelCom), Chi Minh City, pp. 176-181, 2018.

[3] J. M. Williams et al., "Enabling densely-scalable low-power WSNs for shipping and industrial IoT," 2017 IEEE 8th Annual Ubiquitous Computing, Electronics and Mobile Communication Conference (UEMCON), New York, NY, pp. 547-552, 2017.

[4] H. Ayadi, A. Zouinkhi, T. Val, A. van den Bossche and M. N. Abdelkrim, "Network Lifetime Management in Wireless Sensor Networks," in IEEE Sensors Journal, vol. 18, no. 15, pp. 6438-6445, 1 Aug.1, 2018. 
[5] Khan, Junaid Ahmed, Hassaan Khaliq Qureshi, and Adnan Iqbal. "Energy management in wireless sensor networks: A survey." Computers \& Electrical Engineering, 41, 159-176, 2015.

[6] B. T. Nguyen, L. Murphy and G. Muntean, "Energy-Efficient QoS-Based Congestion Control for Reliable Communications in Wireless Multimedia Sensor Networks," 2018 IEEE International Conference on Communications Workshops (ICC Workshops), Kansas City, MO, pp. 1-6, 2018,

[7] H. Jain, R. Jain and S. Sharma, "Improvement of energy efficiency using pdorp protocol in WSN," 2017 International Conference on Information, Communication, Instrumentation and Control (ICICIC), Indore, pp. 1-6, 2017.

[8] Wang, L. \& Xiao, Y. “Mobile Netw Appl” (2006) 11: 723. https://doi.org/10.1007/s11036-006-7798-5.

[9] I. Amdouni, C. Adjih, N. Aitsaadi and P. Muhlethaler, "Experiments with ODYSSE: Opportunistic Duty cYcle Based Routing for Wireless Sensor nEtworks," 2016 IEEE 41 st Conference on Local Computer Networks (LCN), Dubai, pp. 232-235, 2016.

[10] Q. Chen, H. Gao, S. Cheng, X. Fang, Z. Cai and J. Li, "Centralized and Distributed Delay-Bounded Scheduling Algorithms for Multicast in Duty-Cycled Wireless Sensor Networks," in IEEE/ACM Transactions on Networking, vol. 25, no. 6, pp. 3573-3586, Dec. 2017.

[11] Morozs, Nils, Paul Mitchell, and Yuriy V. Zakharov. "TDA-MAC: TDMA without clock synchronization in underwater acoustic networks." IEEE Access 6, 1091-1108, 2018.

[12] Ye, Dayong. "A self-adaptive sleep/wake-up scheduling approach for wireless sensor networks." IEEE transactions on cybernetics" 48, no. 3, 979-992, 2018.

[13] Ayadi, Hayfa, Ahmed Zouinkhi, Boumedyen Boussaid, M. Naceur Abdelkrim, and Thierry Val. "Energy Management in WSN: IEEE 802.15. 4 Unslotted Mode." In 2018 15th International Multi-Conference on Systems, Signals \& Devices (SSD), pp. 1-6. IEEE, 2018.

[14] Mukherjee, Mithun, Yun Rong Lu, Rakesh Matam, and Nikumani Choudhury, "Energy trading in sleep scheduling for wireless rechargeable WSNs," In 2018 10th International Conference on Communication Systems \& Networks (COMSNETS), pp. 429-431. IEEE, 2018.

[15] Zheng, Jun, and Abbas Jamalipour, "Wireless sensor networks: a networking perspective," John Wiley \& Sons, 2009.

[16] Kannan, G., and T. Sree Renga Raja, "Energy efficient distributed cluster head scheduling scheme for two tiered wireless sensor network." Egyptian Informatics Journal, 16, no. 2, 167-174, 2015.

[17] Fang, Wei, Mithun Mukherjee, Lei Shu, Zhangbing Zhou, and Gerhard P. Hancke. "Energy utilization concerned sleep scheduling in wireless powered communication networks," In 2017 IEEE International Conference on Communications Workshops (ICC Workshops), pp. 558-563. IEEE, 2017.

[18] Liu, Feng, Chi-Ying Tsui, and Ying Jun Zhang. "Joint routing and sleep scheduling for lifetime maximization of wireless sensor networks." IEEE Transactions on Wireless Communications 9, no. 7, 2258-2267, 2010.

[19] Ma, Junchao, Wei Lou, Yanwei Wu, X-Y. Li, and Guihai Chen. "Energy efficient TDMA sleep scheduling in wireless sensor networks." In IEEE INFOCOM 2009, pp. 630-638. IEEE, 2009.

[20] W. Ye, J. Heidemann, and D. Estrin, "Medium access control with coordinated adaptive sleeping for wireless sensor networks, " IEEE/ACM Transactions on Networking, vol.12, no.3, pp.493-506, June 2004

[21] W. Ye, J. Heidemann, and D. Estrin, "An energy-efficient MAC protocol for wireless sensor networks," in Proc. IEEE INFOCOM, New York, NY, USA, pp. 1567-1576, Jun. 2002.

[22] Wang, Guijuan, Jiguo Yu, Dongxiao Yu, Haitao Yu, and Li Feng. "Ds-mac: An energy efficient demand sleep mac protocol with low latency for wireless sensor networks." Journal of Network and Computer Applications, 58, 155-164, (2015).

[23] A. Bengheni, F. Didi and I. Bambrik, "Energy-saving comparison of asynchronous MAC protocols for wireless sensor networks," 2017 International Conference on Mathematics and Information Technology (ICMIT), Adrar, pp. 263-268, 2017.

[24] Wong, David, Qian Chen, and Francois Chin. "Directional medium access control (MAC) protocols in wireless ad hoc and sensor networks: a survey," Journal of Sensor and Actuator Networks, 4, no. 2, 67-153, (2015).

[25] Lu, Gang, Bhaskar Krishnamachari, and Cauligi S. Raghavendra. "An adaptive energy-efficient and low-latency MAC for data gathering in wireless sensor networks." In 18th International Parallel and Distributed Processing Symposium, 2004. Proceedings., p. 224. IEEE, 2004.

[26] Kumar, S. Swapna, M. Nanda Kumar, Sheeba VS, and Kashwan KR. "Power Efficient Dynamic MAC Protocol (D-MAC) for Wireless Sensor Networks." Journal of Information \&Computational Science 9, no. 7, 1795-1805, 2012.

[27] Wong, David, Qian Chen, and Francois Chin. "Directional medium access control (MAC) protocols in wireless ad hoc and sensor networks: a survey," Journal of Sensor and Actuator Networks 4, no. 2, 67-153, 2015.

[28] Ren, PinYi, Jia Feng, and YiChen Wang. "A directional MAC protocol with long-range communication ability in ad hoc networks." Science China Information Sciences, 55, no. 4, 765-777, 2012.

[29] Khatarkar, Sarika, and Rachana Kamble. "Wireless Sensor Network MAC Protocol: SMAC \& TMAC." Indian Journal of Computer Science and Engineering (IJCSE) 4, no. 4, 304-310, 2013.

[30] Singh and S. Varma, "An improved TMAC protocol for Wireless Sensor Networks," 2014 International Conference on Signal Propagation and Computer Technology (ICSPCT 2014), Ajmer, pp. 91-95, 2014.

[31] M. I. Khalil, M. A. Hossain, R. Mamtaz, I. Ahmed and M. Akter, "Time Efficient Receiver Oriented Sleep Scheduling for Underwater Sensor Network," 2017 IEEE International Conference on Imaging, Vision \& Pattern Recognition (icIVPR), Dhaka, pp. 1-6, 2017. 
[32] Busch, Costas, Malik Magdon-Ismail, Fikret Sivrikaya, and Bülent Yener. "Contention-free MAC protocols for wireless sensor networks," In International Symposium on Distributed Computing, pp. 245-259. Springer, Berlin, Heidelberg, 2004.

[33] K. Sohrabi and G.J. Pottie, "Performance of a novel self-organization for wireless ad-hoc sensor networks," in Proceedings of the IEEE Vehicular Technology Conference, 1999.

[34] Wang, Long, Yong Liu, and Zengshan Yin. "A Hybrid TDMA/CSMA-Based Wireless Sensor and Data Transmission Network for ORS Intra-Microsatellite Applications." Sensors 18, no. 5, 1537, 2018.

[35] Wang, F.; Kang, Y.; Tan, X.; Yu, K. “A hybrid MAC protocol for data transmission in Smart Grid," In Proceedings of the Conference on Computational Complexity 2014, Portland, OR, USA, pp. 8228-8233, 17-19 June 2015.

[36] Song, W.; Huang, R.; Shirazi, B.; Lahusen, R. "TreeMAC: Localized TDMA MAC protocol for real-time highdata-rate sensor networks," In Proceedings of the IEEE International Conference on Pervasive Computing and Communications, Galveston, TX, USA, 9-13 March 2009.

[37] Durmaz, O.; van Hoesel, L.F.W.; Jansen, P.G.; Havinga, P.J.M. "MC-LMAC: A multi-channel MAC protocol for wireless sensor networks," Ad Hoc Netw., 9, 73-94, 2011.

[38] Rhee, Injong, Ajit Warrier, Mahesh Aia, Jeongki Min, and Mihail L. Sichitiu. "Z-MAC: a hybrid MAC for wireless sensor networks," IEEE/ACM Transactions on Networking (TON), 16, no. 3, 511-524, 2008.

[39] Feng, Juan, and Hongwei Zhao. "Energy-Balanced Multisensory Scheduling for Target Tracking in Wireless Sensor Networks," Sensors 18, no. 10, 3585, 2018.

[40] Wan, Runze, and Naixue Xiong. "An energy-efficient sleep scheduling mechanism with similarity measure for wireless sensor networks," Human-centric Computing and Information Sciences 8, no. 1, 18, 2018.

[41] Yang, Xin, Ling Wang, Jian Xie, and Zhaolin Zhang. "Energy Efficiency TDMA/CSMA Hybrid Protocol with Power Control for WSN." Wireless Communications and Mobile Computing, 2018.

[42] Zhang, Zeyu, Lei Shu, Chunsheng Zhu, and Mithun Mukherjee. "A Short Review on Sleep Scheduling Mechanism in Wireless Sensor Networks," In International Conference on Heterogeneous Networking for Quality, Reliability, Security and Robustness, pp. 66-70. Springer, Cham, 2017.

[43] Mahidhar, Rashmi, and Archana Raut. "A survey on scheduling schemes with security in wireless sensor networks," Procedia Computer Science, 78, 756-762, 2016.

[44] Wang, Di, Mithun Mukherjee, Lei Shu, Yu Chen, and Gerhard Hancke. "Sleep scheduling for critical nodes in group-based industrial wireless sensor networks," In 2017 IEEE International Conference on Communications Workshops (ICC Workshops), pp. 694-698. IEEE, 2017.

[45] Ye, Dayong. "A self-adaptive sleep/wake-up scheduling approach for wireless sensor networks." IEEE transactions on cybernetics 48, no. 3, 979-992, 2018.

[46] Singh, Ripudaman, Brijesh Kumar Rai, and Sanjay K. Bose. "A joint routing and MAC protocol for transmission delay reduction in many-to-one communication paradigm for wireless sensor networks," IEEE Internet of Things Journal 4, no. 4, 1031-1045, 2017.

[47] Kordafshari, M. S., A. Movaghar, and M. R. Meybodi. "A joint duty cycle scheduling and energy aware routing approach based on evolutionary game for wireless sensor networks." Iranian Journal of Fuzzy Systems 14, no. 2, 23-44, 2017.

[48] Fang, Wei, Mithun Mukherjee, Lei Shu, Zhangbing Zhou, and Gerhard P. Hancke. "Energy utilization concerned sleep scheduling in wireless powered communication networks," In 2017 IEEE International Conference on Communications Workshops (ICC Workshops), pp. 558-563. IEEE, 2017.

[49] Baba, S. B., and K. M. Rao. "Improving the network life time of a wireless sensor network using the integration of progressive sleep scheduling algorithm with opportunistic routing protocol," Indian J. Sci. Technol 9, no. 17, 1-6, 2016.

[50] Buratti, Chiara, and Roberto Verdone. "Joint routing and scheduling for centralised wireless sensor networks," In 2016 IEEE 2nd International Forum on Research and Technologies for Society and Industry Leveraging a better tomorrow (RTSI), pp. 1-6. IEEE, 2016.

[51] Chen, Zhuangbin, Anfeng Liu, Zhetao Li, Young-june Choi, and Jie Li. "Distributed duty cycle control for delay improvement in wireless sensor networks." Peer-to-Peer Networking and Applications 10, no. 3, 559-578, 2017.

[52] Oller, Joaquim, Ilker Demirkol, Jordi Casademont, Josep Paradells, Gerd Ulrich Gamm, and Leonhard Reindl, "Has time come to switch from duty-cycled MAC protocols to wake-up radio for wireless sensor networks?." IEEE/ACM Transactions on Networking 24, no. 2, 674-687, 2016.

[53] Oller, Joaquim, Ilker Demirkol, Jordi Casademont, Josep Paradells, Gerd Ulrich Gamm, and Leonhard Reindl. "Wake-up radio as an energy-efficient alternative to conventional wireless sensor networks MAC protocols," In Proceedings of the 16th ACM international conference on Modeling, analysis \& simulation of wireless and mobile systems, pp. 173-180. ACM, 2013.

[54] Deepthi, S. Aruna, E. Sreenivasa Rao, and Giri Prasad. "RTL implementation of image compression techniques in WSN." International Journal of Electrical \& Computer Engineering (2088-8708) 9, no. 3, 2019.

[55] Mahboub, Aziz, El Mokhtar En-Naimi, Mounir Arioua, Hamid Barkouk, Younes El Assari, and Ahmed El Oualkadi, "An energy-efficient clustering protocol using fuzzy logic and network segmentation for heterogeneous WSN," International Journal of Electrical \& Computer Engineering (2088-8708) 9, 2019.

[56] Shiltagh, Nadia A., Mahmood Z. Abdullah, and Ahmed R. Zarzoor. "Evaluation of routing protocol with multimobile sinks in WSNs using QoS and energy consumption parameters." International Journal of Electrical \& Computer Engineering (2088-8708) 9, 2019. 


\section{BIOGRAPHIES OF AUTHORS}

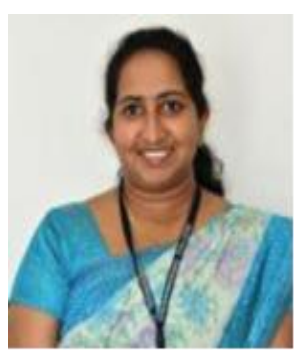

Deepa Mathew K, She is pursuing her Ph.D from Karunya University, Coimbatore, Tamilnadu, India. She has completed her M.Tech in Communication systems Karunya university, Coimbatore, Tamilnadu, India and B.Tech in Electronics and communication Engg ILAHIA College of Engg.\&Tech. Muvattupuzha, Kerala, India. Her areas of interest are Wireless Sensor Networks, Mobile Communication, Digital Image Processing, Power Electronics, GSM, Satellite Communication, Logic Design, Signals and Systems, Optical Fibre Communication and Signals and Systems. She has around 8 years of experience in teaching field.

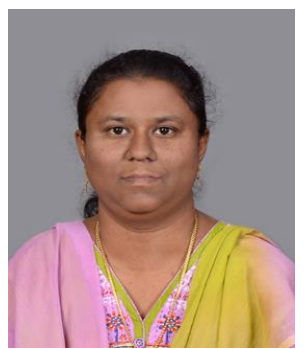

Anita Jones has received B.E degree in Electronics and Communication Engineering from Madurai Kamaraj University, in 1998. She has received M.E degree in Communication Systems from Madurai Kamaraj University, in 2000. She completed her PhD degree in Informationand Communication Engineering under Anna University, Chennai, India in 2014. She is currently working as Assistant Professor in Electronics and Communication Engineering, Karunya University, Coimbatore, India. 